\title{
Enhancing Sustainability Awareness at the University of Technology: Reflections from an On-going Transdisciplinary Research Project
}

\author{
B.O Awuzie , * $^{\text {* }}$ \\ ${ }^{1}$ Department of Built Environment, Faculty of Engineering and Information Technology, Central University of Technology, \\ Bloemfontein, 9300. South Africa
}

\begin{abstract}
This study explores the utility of a transdisciplinary research project framework for improving sustainability awareness levels within a University of Technology. The quest to make contributions to the attainment of society's sustainability aspirations has seen universities transforming into living 'real world' laboratories. Yet, low levels of sustainability awareness among different stakeholders pose a challenge towards institutional transformation. A transdisciplinary research project is proposed as a panacea. Adopting a narrative analysis research design, this study reports on the reflections of different stakeholders concerning the utility of the project and its underpinning framework for the creation of requisite knowledge for efficient building energy management (BEM) and building management (BM) systems -an integral aspect of the research project- directly engenders improved levels of sustainability literacy. Preliminary findings from these reflections indicate the utility of the framework for improving sustainability awareness among stakeholders: academia, support and management staff. Subsequent studies will further reiterate this finding whilst providing a robust methodology for executing similar projects in identical contexts. This paper's originality lies in the use of transdisciplinary research projects to improve sustainability awareness levels across university environments within South Africa
\end{abstract}

\section{Introduction}

The role of universities in knowledge creation has been elucidated [1]. This understanding has led to increased expectations from society for universities to assume leadership roles in championing this paradigmatic shift towards sustainability [2]. Fortunately, universities are increasingly committing to sustainable development (SD) [1-3]. This is evident in the plethora of charters and declarations to which they have signed on to over the past three decades [2]. As such, universities have sought to incorporate SD across all operational aspects, especially teaching and learning, research, procurement, amongst others [4, 5]. Although such efforts have continued to post varied performances, scholars have argued that the implementation efforts have suffered from the compartmentalization mentality which prevails in universities which has, in turn, led to low levels of sustainability awareness among relevant stakeholders therein $[6,7]$. This mentality, propelled by the reductionist nature of the disciplinary-centric culture, has resulted in the prevalence of knowledge/information silos; a salient hindrance to the effective implementation of sustainable development in Universities [8]. As a result, sharing of relevant knowledge concerning SD and its implementation in these institutions has become challenging [6]. This is especially the case in subSaharan African (SSA) countries like South Africa where a strong emphasis on disciplinary-centric engagements persists. Also, this has contributed to the inability of universities to live up to society's expectations in terms of creating and disseminating relevant knowledge as well as engaging with successful sustainability projects which the rest of society can leverage upon [9]. Obviously, for universities in South Africa, optimal SD implementation, as well as subsequent dissemination of lessons learnt from such implementation engagements, remains a significant challenge. Admittedly, some universities have fared above average in SD implementation [7]. But such performances have occurred in a piecemeal, reductionist manner and not in a whole-of-university, systemic manner [10]. Often, the adoption of SD principles stops at the strategic level of universities with the operational staff feigning apathy towards the concept. In other instances, the disconnect between academia and support staff results, not just in the underwhelming incorporation of sustainability ethos into different activities but also knowledge loss from executed projects. Sustainability and SD are complex and multifaceted concepts. And, the implementation of these concepts through projects both within universities and beyond is no exception [11]. Belcher et al. [11] lament the insufficient nature of the extant disciplinary-centric approach to understanding these concepts and their

* Corresponding author: bawuzie@cut.ac.za 
implementation. The fact that different disciplines profess distinct interpretations of what these concepts connote affects the co-production of relevant knowledge required to engender sustainability awareness levels. Therefore, there is a need to engage in the co-production of sustainability-based knowledge in such a manner that it caters to various disciplinary and stakeholder domains present in a university [6]. It is expected that such knowledge will contribute to improved sustainability awareness among stakeholders thereby making for smooth implementation. Unfortunately, a paucity of studies seeking to explore the nexus between knowledge co-production along the higher education institution and SD implementation continuum has been observed. This is despite the challenge posed by the lack of stakeholder buy-in to the implementation process [8]. This observation makes this study imperative. This study seeks to showcase the significance of the transdisciplinary research projects in engendering sustainability awareness among relevant stakeholders in the higher education institutions.

To achieve its objective, this study will report on the author's reflections of the impact of an on-going transdisciplinary research project within a South African UoT (referred henceforth to as SAUoT) in boosting sustainability awareness among various stakeholders at the institution. These reflections will concern the usefulness of this research project's underpinning framework for engendering co-production of sustainability-based knowledge and improved sustainability awareness among stakeholders.

To achieve this objective, this paper will be structured into the following sections: 2. Literature review on transdisciplinary research projects; 3. A description of the nature of the project and the institutional context within which it is situated; 4. A recap on the research methodology utilized; 5. A stepby-step narrative of the steps taken thus far from a transdisciplinary research project perspective; 6. A summary of the preliminary reflections on the study's thematic areas, and; 7. A conclusion.

\section{Literature review}

\subsection{Transdisciplinarity and Knowledge production for sustainable development}

Achieving optimal incorporation of SD ethos across various facets of society's endeavours towards solving the problems associated with sustainability requires the development of relevant cross-cutting knowledge domain [12]. This is especially the case in Higher education institutions where there is increasing advocacy by society for them to champion advancements towards SD and sustainability. Yet, the inability of universities to engage in interdisciplinary research projects has been identified as a barrier to their ability to deliver on this challenge [8]. Research projects can provide veritable platforms for the development of relevant knowledge for tackling global challenges within universities. Also, in reiterating the role of research projects in the creation of such knowledge, [12] point to an assertion from Future Earth stating that "... research will be designed and conducted in partnership with society to produce the knowledge necessary for societal transformations towards sustainability" (420). In furtherance to this, the authors emphasize the need for a paradigmatic shift in the way research is conducted, stressing the salience of collaboration by researchers across disciplinary domains for the co-design of relevant knowledge.

Transdisciplinary research projects have been mooted as one of such probable platforms for securing the involvement of representatives from diverse disciplinary domains within a given context to not only co-produce knowledge but to also at the initial stages, participate in the co-design (formulation) of the problem $[6,11]$. However, forty-years after its introduction in academic literature, there is no widely accepted definition of what transdisciplinary research connotes $[11,14]$. However, there seems to be a consensus among scholars that it is a research approach that stretches beyond the conventional disciplinary boundaries enabling the co-design of the problem by these stakeholders and the co-production of the requisite knowledge to cater to resolving identified problem and change in attitude among stakeholders concerning the issue [12-15]. This observation makes the resort to this approach as a means of creating new knowledge, boosting sustainability awareness among stakeholders and optimally improving implementation performance vis-à-vis the incorporation of sustainability tenets, imperative.

Relevant knowledge for combating issues confronting sustainability and sustainable development has been defined as being mostly context-specific and should reflect nuanced opinions from the constituents of particular contexts to yield desired results. Accordingly, there is a need for transdisciplinary research projects and the project implementation framework as well as the expected contributions thereof to be explored from a multiplicity of perspectives ranging from its utility in knowledge co-production and improvement in sustainability awareness levels among stakeholders.

Based on the foregoing, this study seeks to explain the utility of the transdisciplinary research project (TRP) in engendering improved levels of sustainability awareness among various stakeholder categories within the university. This study will be confined to an aspect of the overall TRP pertaining to the introduction of efficient energy management systems in buildings and building management systems at SAUoT. It does not seek to engage in a discourse pertaining to either of the concepts of sustainability/SD or the role of universities in fostering these concepts as a lot of literature already exists in that wise.

\subsection{Description of Institutional and Project Context}

SAUoT happens to be at the forefront of the transformation towards a sustainable and smart future. This is evident in the marketing communication 
documents as well as mission statements emanating from the institution. In furtherance to this, the institution is aware of its relationship with the wider society, herein represented by the city-wide geographical domain within which its campuses are located, firstly as a centre for knowledge production and dissemination, secondly as a hub for skills development and advancement, thirdly as a living laboratory for experimenting on probable solutions to city-wide challenges and, finally as a platform for futuristic agenda-setting for the citywide initiatives. Accordingly, SAUoT has made known its intention to transform, at first instance, into a sustainable and smart university of technology and subsequently utilize the experience garnered in championing city-wide/region-wide transformation towards a sustainable and smart future. Over the years, this aspiration has met with various encumbrances, most of which can be categorized as organizational factors [16]. Of significance among these factors are knowledge-related organizational factors. Factors under this category result from the disciplinary-orientated nature of the university wherein a sharp dichotomy between the academic disciplines on hand and between the academic and support disciplines, on the other hand, serves to hinder incorporation of sustainability ethos into SAUoT's activities. This has brought about this TRP and TRF initiative to boost collaborative development of relevant knowledge.

Rising energy costs happens to be a major constraint affecting contemporary societies in the global south and the global north. The rising incidence of energy poverty across the world is a pointer to the prevalence of this constraint. This constraint is not peculiar to individuals, households or communities but also different organizations-both public and private sector owned entities respectively. The entities are continually seeking ways to overcome this challenge hence the increasing resort to alternative means of power and the development of a cocktail of off-grid energy sources. Efforts are being made presently to curb energy/power wastages or losses through the adoption of different retrofitting measures and practises. The case at SAUoT is no different. With a vast holding in property assets comprising of student halls of residence, lecture theatres and administrative office blocks, energy consumption levels and cost associated with it are expected to go through the roof. As such, tackling such incidences through the adoption and implementation of efficiencysavings orientated procedures such as BEMS and BMS has become a priority.

Yet, these measures bother heavily on the use of software and hardware packages to moderate energy consumption. The use of sensors, retrofitting of existing structures, change in lighting systems towards energy efficient systems such as light emitting diodes (LED) have resulted from this programme. But, these interventions are being carried out in a piecemeal manner, along with disciplinary lines with no attempt to capture and transfer knowledge from lessons learnt across a transdisciplinary plane. Furthermore, relevant stakeholders feign ignorance of the interventions being made by other parties towards this aspiration. This accentuates the commentary highlighted before on the need for collaborative production of relevant sustainability knowledge to tackle such challenges. In their study, Mohammed et al. [17] posit the salient need to incorporate the 'heartware' into the softwarehardware continuum upon which the implementation of energy efficiency initiatives in organizations is predicated. According to them, the heartware component comprises of the socio-aspect of the sociotechnical system, i.e. the individuals and the relationship existing between them and the influence of such relationships on the prevailing culture within a given environment.

The approach culminated in the evolution of a transdisciplinary research project on effective energy management in buildings at the SAUoT. This study proceeds to report on the author's reflection on the utility of the project and the framework in advancing sustainability awareness among stakeholders at SAUoT and facilitating the attainment of the institution's sustainable and smart campus aspirations.

\section{Research Method}

A narrative analysis research design has been adopted in this study. This design comes naturally to the study considering the impetus it gives to researchers who are trying to make sense of everyday lived experiences of either themselves or other distinct actors through stories [18-20]. This design shares some similarity with phenomenology when done at a personal level, but differs when executed at interpersonal, positional or ideological levels [18]. In this case, the author relies on his series of interactions with different stakeholder groups at different intervals on the TRP to reflect on the influence of the TRP and the TRF on their understanding of efficient energy management in buildings through different interventions, the possible contributions that they can make based on the genre of scientific episteme they postulate as the skills that they possess and their understanding of the nexus between the phenomenon and sustainable development across multiple scales. Considering its qualitative nature, data was collected through a juxtaposition of the data collection techniques such as unstructured interviews, participant observations, and document reviews. Memos were created during these interactions and subsequently re-read, in the aftermath, severally to enable sensemaking based on the pre-set themes guiding the analysis. These memos also assisted the reflection phase immensely. Participants were recruited from two distinct categories of stakeholders: academia and support staff from the relevant Department-Estate and Infrastructure (E\&I) at SAUoT. Also, the academic staff members were drawn from different disciplines present in the institution. At this initial phase, interaction with students is not reported. In the next section, a review of the steps taken thus far in this TRP as indicated by a TRF is presented. 


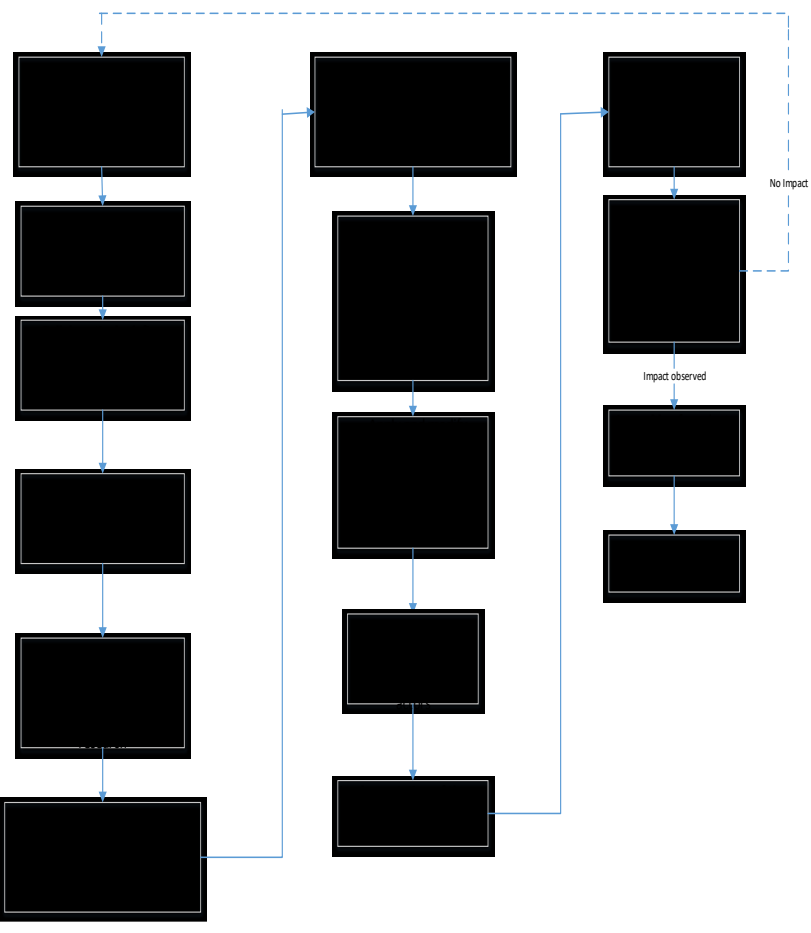

Fig. 1. Proposed Transdisciplinary Research Framework (Adapted from Jahn et al. 2012, Mauser et al. 2013, Gaziulusoy and Boyle, 2013, Schäpke, et al. 2018)

\subsection{Project initial stages}

The execution of a TRP relies on the robustness of a TRF to achieve the desired objectives. The TRF upon which this study was premised is shown in Figure 1. A rendition of the processes and the role of the researcher therein are explicated in the following sections.

\subsubsection{Phase 1: Problem transformation}

Actor and context analysis: This analysis was carried out to determine the relevant actors influenced, influence or like to influence the execution of the BEMS at the University. In this case, the researcher understood his environment: the SAUoT context and as such, engaged in a review of the Sustainable Development Implementation Framework (SDIF) document to itemize the activities listed therein for efficient energy management in institutional buildings and the parties responsible. This enabled an adept identification of the actors therein.

Collaborative definition of real-world problems and goals to be addressed: Driven by these findings, a brainstorming session which doubled as a focus group was convened with representatives of various stakeholders within the university community. Accordingly, students, academic staff from the 4 faculties, support staff from departments like finance, procurement, facilities, registry were invited to the focus group. The aim of this session was to elicit the perspectives of these stakeholder categories concerning the issues on various matters influencing the institutional goals of sustainable smart campus status, with emphasis on various aspects inclusive of energy management arising from the deployment of the internet of things platform among others. Yet, this study will only seek to take forward the aspect concerning energy management in buildings. Accordingly, follow-up meetings were held with a smaller number of stakeholders including the researchers to decide on projects that can be executed to achieve efficiencysavings in energy management.

Development of research questions: This stage involved the setting up of different meetings at different intervals. During these workshops, opportunities will be provided for different stakeholder groups including experts from the academic disciplines to interrogate the institutional problems on efficient energy management formulated in step 1.1. The focus of these sessions was fixated on the institutional problem framed previouslyi.e. efficient energy management. These sessions provided a context for team formation between members of these groups based on their perspective of the problem being discussed. A central research question: 'How can we engage with the management of energy utilization in the buildings on campus?' was developed. This research question led to the development of other sub-research questions like:

1. 'What are the relationship between efficient energy management in SAUoT and the institution's sustainable and smart university aspirations?';

2. 'What factors are driving the current energy utilization regime at SAUoT?

3. 'How can smart technologies be adopted and applied toward the attainment of this aspiration?';

4. 'What engineering/sociological solutions are available for achieving this feat and how can they be integrated?'

5. 'What roles are available for different stakeholders in enabling the implementation of alternative energy management protocols at SAUoT to achieve the desired-levels of efficiency savings?'

The composition of a Collaborative research project team: Based on the research questions developed, the researchers carried out an analysis of the skill set required to provide answers to these questions with a bid to ascertain if any of the desired skills/competencies were lacking and probably recruit such skills. This research project is currently at this stage of team selection and will only report on reflections up to this point.

Designing framework for collaboration for knowledge co-production: In this phase, integral parts of the SDIF as it concerns energy management will be deployed in building a collaborative framework for the co-production of sustainability-related knowledge for effective among the stakeholders identified and recruited initially.

\subsubsection{Phase 2: Co-Production of Knowledge}

Assembly and assignment of roles to stakeholders: In this stage, the researchers will allocate roles to the 
recruits in accordance with their competencies within each of the sub-units developed based on the designed framework. Some criteria for allocation will be developed prior to this time by the researchers based on the project goals and expected impact on participants. Yet care will be taken to have researchers working with each of the teams to document the knowledge that was being co-produced within the teams.

Application and modification of methods: In the proposed project, the SDIF will serve as the implementation framework whereas the working group will be expected to dissolve into groups based on competencies as described in 2(a). However, the grouping and the methods applied within the groups can be adjusted upon reviews of performance.

Development of tangible boundary concepts for all actors: This is done according to the competencies possessed by the participants (actors) and the expected transformation expected from them at the end of the process.

Implementation of the integration platform: At this phase, researchers assigned to the smaller units working on different aspects of the SDIF will be required to transmit new knowledge which will be co-produced because of the execution of the assigned tasks to the investigators. The members of the task team will be expected to discuss how they may have benefitted from their working experiences with experts from other disciplines and how they think that other experts have benefitted from their own expertise. This will be documented by the researchers.

\subsubsection{Phase 3: Transdisciplinary integration}

Assessment of integrated results: This phase will involve the collection of all the memos recorded and transcripts from the sub-group interactions and the perceptions of the participants of their experiences whilst working on the projects. This information will subsequently be reviewed and evaluated by the researchers with the aid of external parties who acted as workshop facilitators during step 1 .

The evaluations will be predicated on the change in the level of sustainability awareness for an individual participant, the degree of appreciation of the place of interdisciplinarity and transdisciplinarity in undertaking SD projects. And above all, the performance of the SDIF based on the quality of expected and delivered outcomes. But, a feedback loop is provided if the majority of the participants did not indicate any significant improvement in their mindsets and degree of sustainability literacy. This will imply a problem with the framework, hence necessitating a review of the problem formulation stage.

If improvements were noticed across the facets, then the researchers expect to provide a detailed artefact of the pathway undertaken in bringing about these improvements and present same as a guidance document for facilitating the attainment of project goals around the SDIF in South African Universities.

\section{Preliminary Reflections}

As previously stated, this study is at an embryonic stage and can only report on reflections pertaining to happenings at the initial stages of the TRP as explained through the TRF. Thus far, the study will report on the thematic area underpinning the entire study, namely: The Influence of TRF on co-production of relevant knowledge for efficient energy management by relevant stakeholders and any improvement of sustainability awareness levels among these stakeholders. The reflections on the latter are based on their (stakeholder's) appreciation of the extant nexus between efficient energy management of the institution's buildings and the institution/city/region/global sustainable and smart city aspirations.

Arising from the interactions with various actors through the various platforms available to the author in various stages of step 1 of the TRF, it was easy to see a shift in the conventional disciplinary approaches to solving sustainability-orientated problems towards a more inter-, and transdisciplinary approach. A better appreciate the concept of sustainable development and smartness by the stakeholders is presently being felt along the implementation continuum. Ostensibly, the plethora of meetings and the focus group sessions created a platform for elicitation of perspectives and countenance of fears held by stakeholders from different scientific epistemes relating to the impact of sustainable development and smartness on their disciplines ad day jobs. Also, solutions such as the use of smart technologies underpinned by the Internet of Things (IoT) have started being proffered by experts working in that area. Solutions that bother on the behavioural tendencies of occupants are also being brought forward thus ensuring that no stakeholder group is left behind. This implies the initial steps to the co-production of relevant knowledge for energy efficiency in university buildings/campuses.

The reflection is at this stage limited by the present stage of the TRP. But in future studies, efforts will be made to comprehensively articulate the shifts in perception and knowledge base of different actors to the energy efficiency TRP and the impact of this on the coproduction of relevant knowledge whilst boosting the levels of sustainability awareness among stakeholder who were hitherto unaware of the nexus and thus undermining the implementation processes on campus.

\section{Conclusion}

Based on available evidence, the role of universities in championing society's sustainability aspirations is being called into question in recent times. This is due to the unsustainable consumption patterns which have remained prevalent across various universities, especially in the Global South. Business, as usual, remains more of a norm in these contexts as universities therein appear to be paying lip service to the sustainable development agenda. Low levels of sustainability 
awareness among stakeholders have been identified as a salient contributor to this notion.

Poised to assume a sustainable and smart university status, a South African University of Technology has set out to explore the utility of a transdisciplinary research framework (TRF) in facilitating an improvement in the sustainability awareness levels witnessed in that institution. This study reports on the nature of the TRF and the steps situated within it. As such, it utilizes a TRP on efficient energy management at a SAUoT to illustrate the utility of the TRP and TRF in engendering improved sustainability awareness it highlights the expected outcomes from each phase of the TRF-three main phases were proposed. It is expected that the full operationalization of the TRF will bring about reduced apathy concerning sustainability and SD by implementation personnel, either within the academia, students, or support staff stakeholder categories through their incorporation in problem-solving and knowledge co-production. This study can best be described as being at an embryonic stage but with immense implications for universities desirous of improving sustainability awareness levels through TRPs. Expectedly, further studies will present experiences garnered from the actual TRF operationalization within the UoT context as well as its impact on extant levels of sustainability awareness therein.

\section{References}

1. W. L. Filho, About the role of universities and their contribution to sustainable development. Higher Education Policy, 24(4), pp. 427-438, (2011)

2. R. Lozano et al., Declarations for sustainability in higher education: becoming better leaders, through addressing the university system. Journal of Cleaner Production, 48, pp. 10-19, (2013)

3. W. L. Filho, Sustainability at universities: Opportunities, challenges and trends, 2009).

4. B. Awuzie, F. Emuze, and A. Ngowi, Towards a Social Ontology on Sustainable Development in CUT: Understanding Stakeholder Perceptions, in Handbook of Theory and Practice of Sustainable Development in Higher Education, W. Leal Filho, et al., Editors. Springer: Cham, Switzerland, pp. 425-439, (2017).

5. T.B. Ramos et al., Experiences from the implementation of sustainable development in higher education institutions: Environmental management for sustainable universities. Journal of Cleaner Production, 106, pp. 3-10, (2015).

6. M. Adomssent, J. Godemann, and G. Michelsen, Transferability of approaches to sustainable development at universities as a challenge. International Journal of Sustainability in Higher Education, 8(4), pp. 385-402, (2007)

7. R. Lozano et al., A review of commitment and implementation of sustainable development in higher education: results from a worldwide survey. Journal of Cleaner Production, 108, pp. 1-18, (2015)

8. Velazquez, L., N. Munguia, and M. Sanchez, Deterring sustainability in higher education institutions: An appraisal of the factors which influence sustainability in higher education institutions. International Journal of Sustainability in Higher Education, 6(4), pp. 383-391, (2005)

9. S. Sedlacek, The role of universities in fostering sustainable development at the regional level. Journal of Cleaner Production, 48, pp. 74-84, (2013).

10. J. McMillin, and R. Dyball, Developing a wholeof-university approach to educating for sustainability linking curriculum, research and sustainable campus operations. Journal of Education for Sustainable Development, 3(1), pp. 55-64, (2009)

11. B.M. Belcher et al., Defining and assessing research quality in a transdisciplinary context. Research Evaluation, 25(1), pp. 1-17, (2016).

12. W. Mauser et al., Transdisciplinary global change research: the co-creation of knowledge for sustainability. Current Opinion in Environmental Sustainability, 5(3-4), pp. 420431, (2013).

13. R.J. Lawrence et al., Promoting decent work in the construction sector: The role of local authorities. Habitat International, 32(2), pp. 160-171, (2008).

14. T. Jahn, M. Bergmann, and F. Keil, Transdisciplinarity: Between mainstreaming and marginalization. Ecological Economics, 79, pp. 1-10, (2012).

15. J. Zscheischler, S. Rogga, and M. Busse, The adoption and implementation of transdisciplinary research in the field of landuse science-a comparative case study. Sustainability, 9(11), pp. 1926, (2017).

16. B. Awuzie, and F. Emuze. An identification of organizational factors affecting sustainable development in a South African University. in Proceedings of the 4th Construction Management Conference, Port Elizabeth, South Africa, (2015).

17. Z. F. Mohamad et al., Heartware as a Driver for Campus Sustainability: Insights from an Action-oriented Exploratory Case Study. Journal of Cleaner Production, 196(September 2018), pp. 1086-1096, (2018).

18. C. Stephens and M. Breheny, Narrative analysis in psychological research: An integrated approach to interpreting stories. Qualitative Research in Psychology, 10(1), pp. 14-27 (2013).

19. C., Emden, Conducting a narrative analysis. Collegian, 5(3), pp. 34-39, (1998). 
20. S. Earthy and A. Cronin, Narrative analysis, in Researching Social Life N. Gilbert, Editor. Sage:London, (2008) 